\title{
Er:YAG laser debonding of porcelain laminate veneers
}

\author{
Yong Zhang' ${ }^{1}$, Jean-Paul Rocca ${ }^{1,2}$, Carlo Fornaini' ${ }^{2}$, Yinghua Zhen ${ }^{1}$, Zengyi Zhao', Elisabetta \\ Merigo $^{2}$ \\ 1 Shijiazhuang $2^{\text {nd }}$ Hospital, Dpt of Stomatology, Shijiazhuang (Hebei) China. \\ 2 Micoralis laboratoy EA 7354, University Côte d'Azur, Nice, France \\ * Correspondence: Prof. Elisabetta Merigo - Micoralis Laboratory EA7354 - University Côte d'Azur, Nice, \\ France - E-Mail: elisabetta.merigo@unice.fr
}

\begin{abstract}
The purpose of this study consists to verify if Er:YAG laser, at low fluences, is able to debond porcelain veneers, successfully used to improve anterior tooth esthetics, without damaging the tooth structures.

A total of twelve freshly extracted teeth were used and samples were decontaminated, stored, prepared to obtain Veneers adhesion. One week after, Er:YAG laser with a non-contact sapphire tip with air-water spray was used for veneer debonding at $100 \mathrm{~mJ}$ of power and $30 \mathrm{~Hz}$ of frequency (Fluence $5.91 \mathrm{~J} / \mathrm{cm}^{2}$ ).

Results demonstrated that veneer debonding is possible with an Er:YAG laser and that the total number of pulses does not seem related to its efficiency. SEM observation confirms that residual tooth structure is not altered when using these low fluences.

Low fluences with Er:YAG laser are able to debond veneers while preserving the tooth structures. SEM observation confirmed that residual tooth structure is not altered with low fluences.
\end{abstract}

Keywords: er:yag laser; debonding; laminate veneer; scanning electron microscopy

\section{Introduction}

The use of ceramic laminate veneer has considerably and successfully grown to improve anterior tooth esthetics in recent years [1,2]. The interest of these restorations is attributable to their conservative preparation, aesthetic quality, discoloration (resin cements), fracture resistance, tissue acceptance, low debonding rate, negligible incidence of caries and patient satisfaction [3].

The long-term success of these kinds of restoration seems to be in function of an adhesive cementation and, for this purpose, several methods for crown and teeth preparation has been proposed [4].

As such all dental restorations, ceramic veneers have limited lifespan, and may ultimately need replacing at variable intervals [5]. Successful debonding process relies on maintaining the enamel structure without producing iatrogenic damage, so allowing the enamel surface restoration, as closely as possible [6,7].

In addition, replacement of failed restorations may be time-consuming and also compromise the additional tooth structure [8]. Bishara et al., demonstrated that excessive debonding strength may cause enamel cracks [9].

To reduce the irreversible enamel surface damage, several methods of ceramic veneers debonding have been suggested. Vertical and horizontal grooves can be made using a tapered diamond bur and the ceramic fragments removed with a flat plastic or an ultrasonic instrument [10].

Moreover, advancements in laser technology have led to multiple dental applications, such as orthodontics [11-13]. One of these lasers, Erbium-doped, Yttrium-Aluminum-Garnet (Er:YAG), has been successfully used for debonding ceramic brackets. 
The removal of ceramic laminate veneers with laser is reported only in a scanty number of publication and for this reason the importance and the aim of this ex vivo study consist to verify the ability of Er:YAG laser for laminate veneers debonding with the preserving of the tooth structures (SEM observations).

\section{Materials and Methods}

A total of twelve freshly extracted teeth for periodontal reasons were used in this study. Patients were informed about the inclusion of their extracted teeth in a clinical study and their consent was registered, according to the local ethic committee.

Samples were decontaminated, stored in a $0.1 \%$ thymol solution for two days and rinsed with water and crowns were then prepared as follows:

- Enamel preparation: vestibular enamel surfaces were prepared under air water spray with a turbine (Kavo Supertorque 660 B, Germany), diamond burs (Dental Diamond bur, Mani Dia Burs, TR26, 26F Japan) and polished with TR26EF burs.

- Impression: once macroscopic evaluation of the preparation was recognized as acceptable, impression were realized in the laboratory (Polysiloxane duplication material, Correcsil, Yamahachi dental MFG, Co, Japan), then porcelain veneers were realized commonly (powder Duceram Kiss, USA).

- Sealing: the sealer used was Universal adhesive (Single bond 3M, Espe, USA) and the cement was the Veneer cement (RelyX Veneer 3M Espe, USA). After the realization of the veneers, their thickness was checked at three locations (incisal edge, middle third and cervical third).

Teeth were then kept in closed Eppendorf tubes for one week on a humid atmosphere. One week after, Er:YAG laser (Lite Touch, Syneron, Israel) with a sapphire tip (diameter $0.8 \mathrm{~mm}$, length 14 $\mathrm{mm}$ ) in a non-contact mode (working distance of 1 to 2 millimeters) with an abundant air-water spray (4/4 ratio) was used for veneer debonding.

Settings of $100 \mathrm{~mJ}$ and $30 \mathrm{~Hz}$ were chosen corresponding to a fluence of $5.91 \mathrm{~J} / \mathrm{cm}^{2}$. The total number of pulses was recorded at the beginning and at the end of the irradiation and, by their difference, the pulses necessary to take off the veneer were calculated. A mean range plus a standard deviation was calculated (twelve samples) (Table 1).

Once samples have been removed, coronal surfaces and ceramic laminate veneers were coated with a thin film of gold $(\mathrm{Au})$ in a vacuum evaporator (Ion Sputter, JEOL, Japan) and observed under a scanning electron microscope (JEOL JSM-5310LV, Japan) in low vacuum mode between 15 and 20 $\mathrm{kV}$. Images were processed for display using SemAforE software (JEOL AB, Japan).

\section{Results}

All veneers were completely and easily removed from the tooth using the Er:YAG laser. The pulse number, registered automatically by the device, ranged from 17157 to 4077 (mean range 9836,25) (Table 1 ) and great standard deviation value was registered $(4501,91)$. The average removal time was 328 seconds (Standard deviation 156 seconds), while the removal time ranged from 136 to 572 seconds.

SEM observation (Figures 1-4) confirms that residual tooth structure is not altered when using these low fluences.

In addition, the removal occurred without ablating or damaging any tooth structure according to SEM observations.

\subsection{Figures and Tables}




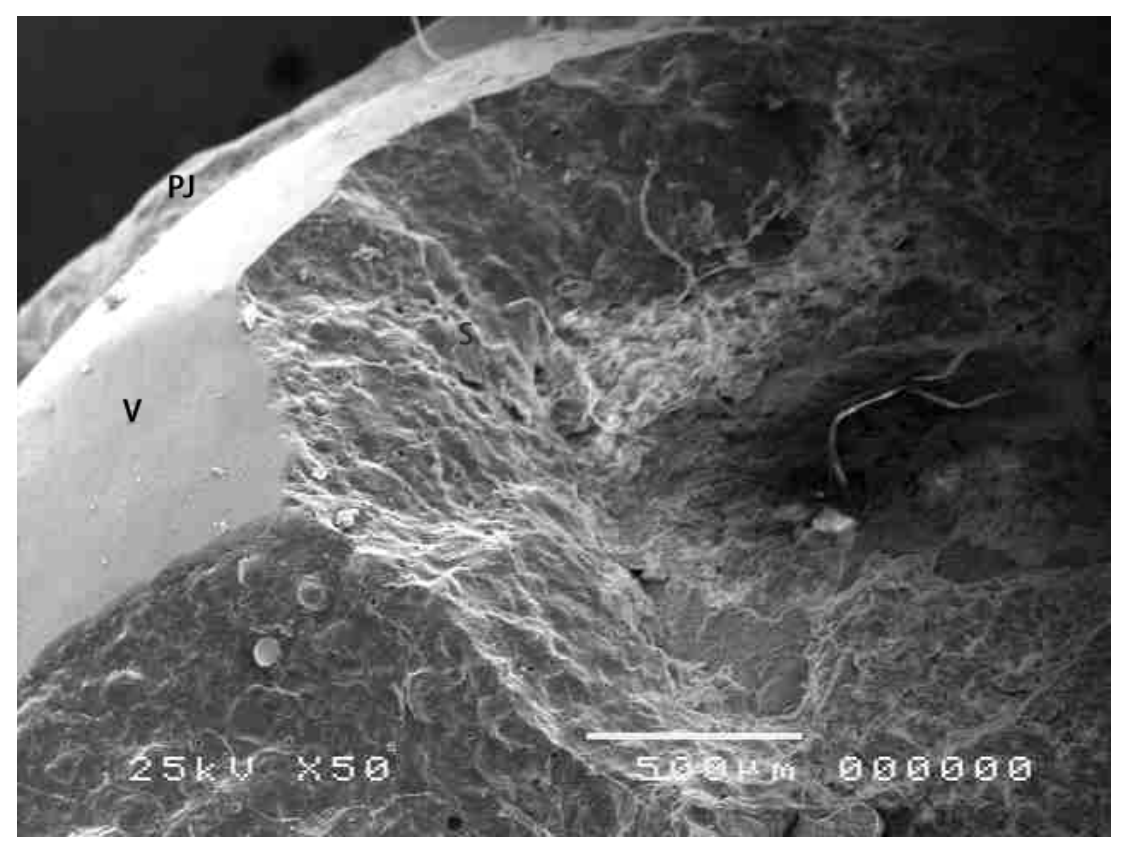

Figure 1. Broken veneer after irradiation (PJ: Peripheral Joint; V: Veneer broken; S: Sealer).

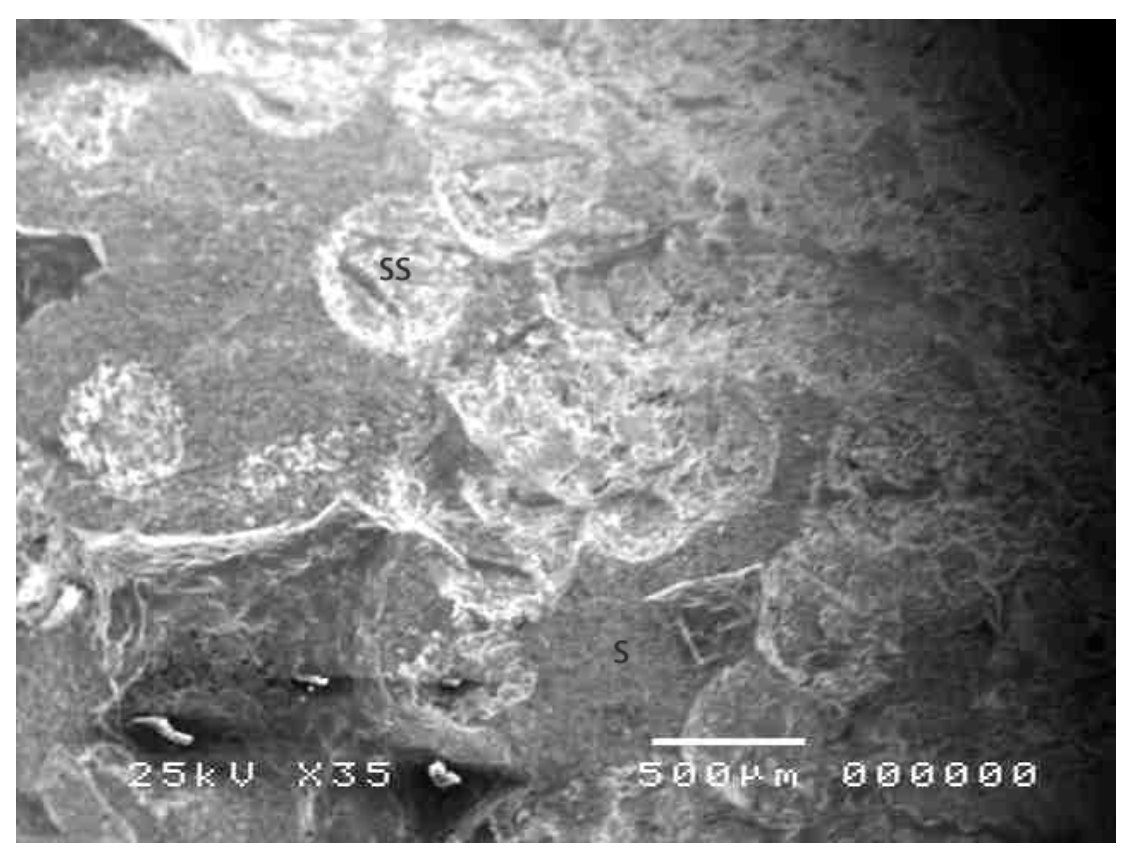

Figure 2. Broken veneer (V), Sealer (S) and Spot Size (SS) on tooth surface. 


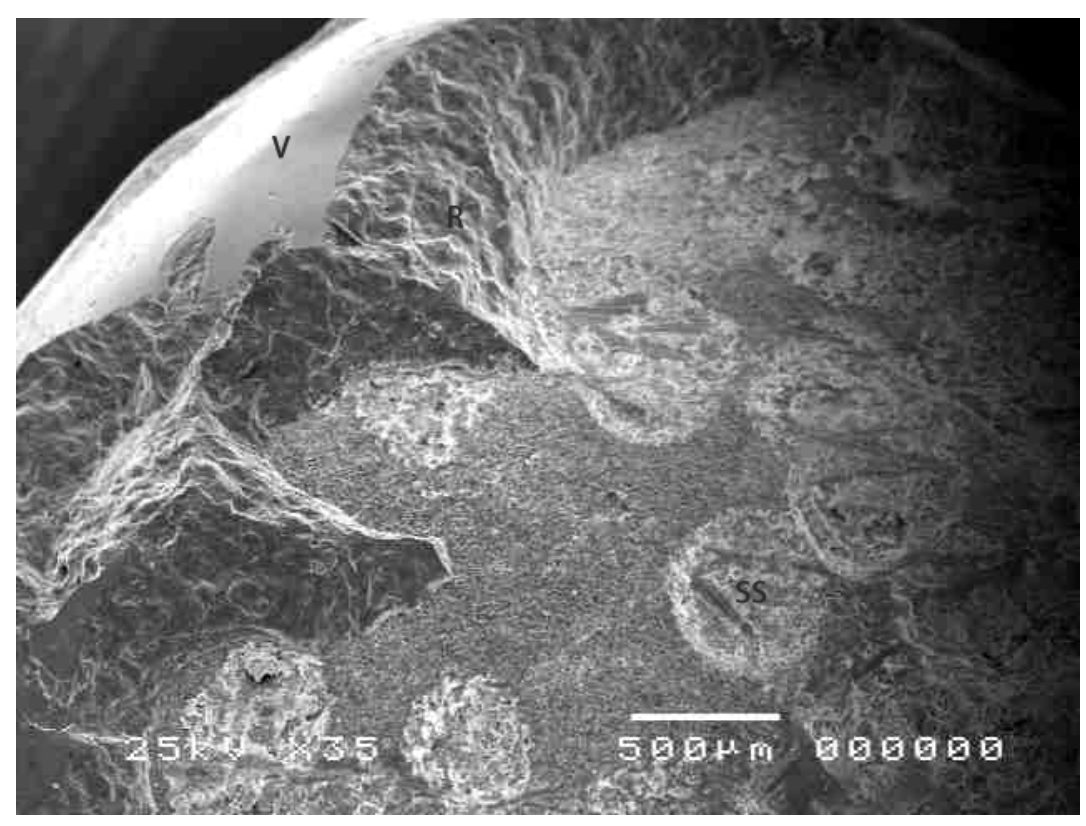

Figure 3: Veneer debonding: Spot Size (SS) of about $0.5 \mathrm{~mm}$ corresponding approximately to the diameter of the laser sapphire tip.

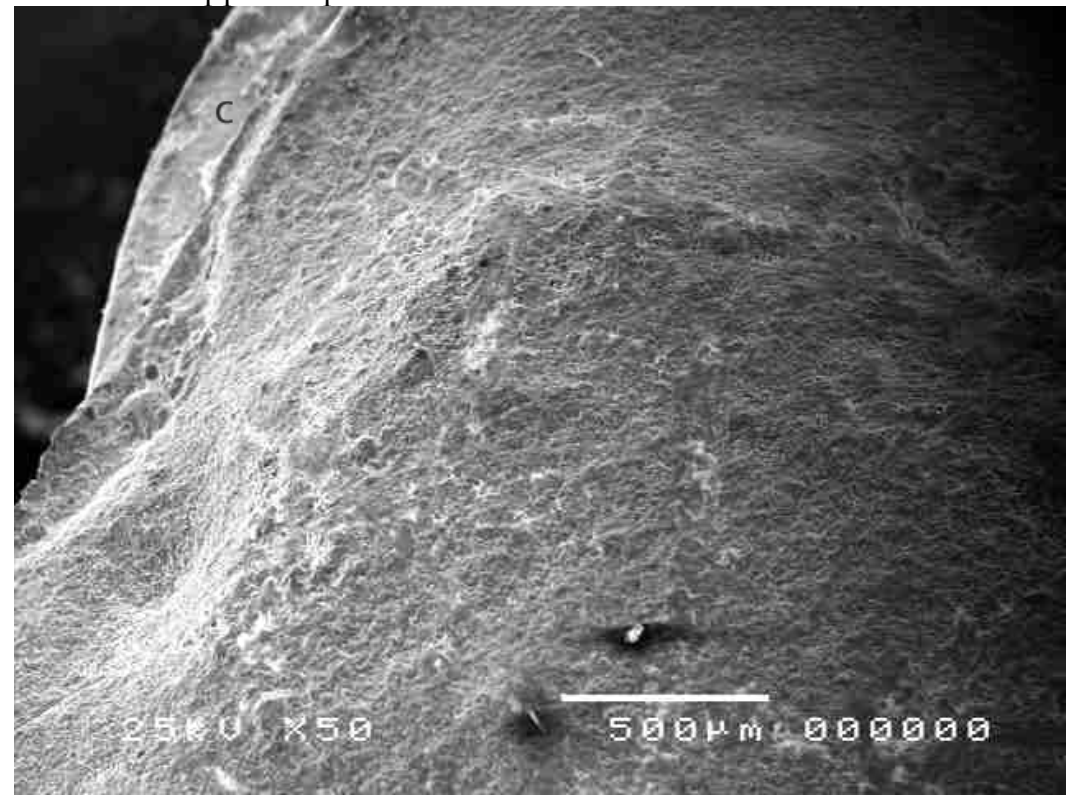

Figure 4: Peripheral preparation with chamfer bur (C) and debonded veneer. 
Table 1. Number of pulses and total working time (seconds) to remove every veneers, Mean and Standard deviation.

\begin{tabular}{|c|c|c|}
\hline Sample & Number of pulses & $\begin{array}{c}\text { Total working time } \\
\text { (sec) }\end{array}$ \\
\hline 1 & 4077 & 136 \\
\hline 2 & 10417 & 347 \\
\hline 3 & 6431 & 214 \\
\hline 4 & 14938 & 498 \\
\hline 5 & 10389 & 346 \\
\hline 6 & 17157 & 572 \\
\hline 7 & 6717 & 224 \\
\hline 8 & 6672 & 222 \\
\hline 9 & 16635 & 555 \\
\hline 10 & 11902 & 397 \\
\hline 11 & 5052 & 168 \\
\hline 12 & 7648 & 255 \\
\hline Mean & 9836,25 & 328 \\
\hline SD & 4501,91 & 156 \\
\hline
\end{tabular}

\section{Discussion}

In these experimental conditions all the irradiated veneers were debonded, eight of them entirely, four into two or three parts: the removal occurred without damaging any tooth structure according with SEM images.

To avoid fracturing the veneer during laser debonding could be an advantage if the veneer had been misplaced and needs to be repositioned, as confirmed by Monford and coworkers [14] and the optimization of the used parameters may allow us to obtain this result in the totality of debonding procedures.

A great standard deviation value was registered and this is very difficult to explain, due to multiple parameters involved. Even if one of the possible biases has been surely avoided by the utilization of the same operator for all the tests, width of the samples, quality of the cementation, time to reach the peak power after each stop and restart are probably all involved, and, unfortunately, their respective influence on veneer debonding is unknown.

It is to underline that, when, in the literature it is affirmed that the time for debonding with quite the same parameters does not exceed 4 minutes, in this evaluation it is necessary to distinguish between working time and irradiation time that, when using pulsed lasers, are very different (duty cycle). As an example, pulse duration of $800 \mu \mathrm{sec}$. corresponds to $8.10^{-4} \mathrm{sec}(0.0008 \mathrm{sec})$ and, for a frequency of $30 \mathrm{~Hz}$, the irradiation time per second becomes $30 \times 0.0008 \mathrm{sec}(0.0024 \mathrm{sec}$.) while the resting time becomes $1-0.0024=0.99 \mathrm{sec}$. 
The irradiation time was extremely short to cross the underside surface of the porcelains veneers and the relaxation time so long, when compared to the irradiation time.

On such biomaterials, there is no evidence of a relationship between relaxation time and absence of cracks because, theoretically, there is only a poor absorption of this wavelength in these kind of ceramics. The explication of the interaction may be linked with the presence of silica and polymethyl metacrylate resin [14], even in few quantities, in the dental ceramics and they are able to absorb this wavelength.

The veneer debonding by the use of Er:YAG laser allows the dentist to re-use the detached veneer in some clinical cases when preservation of this fixed prosthetics integrity is observed.

In the literature it was previously confirmed that veneers do not show any water absorption while the bonding cement (Relyx) showed a broad $\mathrm{H}_{2} \mathrm{O} / \mathrm{OH}$ absorption band [14] and initial signs of cement ablation starts with very low fluences. In our observation we confirmed that low fluences were sufficient to debond veneers.

Author Contributions: Conceptualization, J.P.R., Y.Z. and Z.Z.; Methodology, J.P.R.; Software, Y.Z. and Y.Zhe.; Investigation, Y.Z. and Y.Zhe.; Data Curation, E.M. and C.F.; Writing-Original Draft Preparation, J.P.R, E.M. and C.F.; Writing-Review \& Editing, E.M. and C.F.

Funding: "This research received no external funding".

Conflicts of Interest: “The authors declare no conflict of interest."

\section{References}

1. McLaughlin G. (1998) Porcelain veneers. Dent Clin North Am 42:653-6.

2. Blatz M.B. (2002) Long-term clinical success of all-ceramic posterior restorations. Quintessence Int 33:415-26.

3. Guruprasad A., Rivankar N., Dhiman R.K., Viswambaran M. (2015) Evaluation of the effect of surface preparation using phosphoric acid and luting cement on the flexural strength of porcelain laminate veneering material.Med J Armed Forces India 71:299-305.

4. El Gamal A., Fornaini C., Rocca J.P., Muhammad O.H., Medioni E., Cucinotta A., Brulat-Bouchard N. (2016) The effect of CO2 and Nd:YAP lasers on CAD/CAM Ceramics: SEM, EDS and thermal studies. Laser Ther 25:27-34.

5. Whitehead S.A., Aya A., MacFarlane T.V., Watts D.C., Wilson N.H. (2000) Removal of porcelain veneers aided by a fluorescing luting cement. J Esthet Dent 12:38-45.

6. Shuler F.S., Van Waes H. (2003) SEM-evaluation of enamel surface after removal of fixed orthodontic appliances. Am J Dent 16:390-4.

7. Ozer T., Basaran G., Kama J.D. (2010) Surface roughness of the restored enamel after orthodontic treatment. Am J Orthod Dentofac Orthop $137: 368-74$.

8. Magne P., Kwon K.R., Belser U.C., Hodges J.S., Douglas W.H. (1999) Crack propensity of porcelain laminate veneers: a simulated operatory evaluation. J Prosthet Dent 81:327-334.

9. Bishara S.E., Fonseca J.M., Boyer D.B. The use of debonding pliers in the removal of ceramic brackets: force levels and enamel cracks (1995) Am J Orthod Dentofac Orthop 108:242-8.

10. McCullock A.J. (1992) Dental demolition. Dent Update 19:255-262.

11. Tocchio R.M., Williams P.T., Mayer F.J., Standing K.G. (1993) Laser debonding of ceramic orthodontic brackets. Am J Orthod Dentofacial Orthop 103:155-62.

12. Strobl K., Bahns T.L., Willham L., Bishara S.E., Stwalley W.C. (1992) Laser-aided debonding of orthodontic ceramic brackets. Am J Orthod Dentofacial Orthop $101: 152-8$.

13. Nalbantgil D., Oztoprak M.O., Tozlu M., Arun T. (2011) Effects of different application durations of ER: YAG laser on intrapulpal temperature change during debonding. Lasers Med Sci 26:735-40.

14. Morford C.K., Buu N.C., Rechmann B.M., Finzen F.C., Sharma A.B., Rechmann P. (2011) Er:YAG laser debonding of porcelain veneers. Lasers Surg Med 43:965-74. 